\title{
Magnetically Directed Targeting Aggregation of Radiolabelled Ferrite Nanoparticles
}

\author{
Yuh-Feng Wang, ${ }^{1,2,3}$ Chao-Ming Fu, ${ }^{4}$ Mei-Hua Chuang, ${ }^{5}$ Thau-Ming Cham, ${ }^{3}$ \\ and Mei-Ing Chung ${ }^{3}$ \\ ${ }^{1}$ Department of Nuclear Medicine, Buddhist Dalin Tzu Chi General Hospital, Chiayi 62247, Taiwan \\ ${ }^{2}$ School of Medicine, Tzu Chi University, Hualien 97004, Taiwan \\ ${ }^{3}$ School of Pharmacy, Kaohsiung Medical University, Kaohsiung 80708, Taiwan \\ ${ }^{4}$ Department of Physics, National Taiwan University, Taipei 10617, Taiwan \\ ${ }^{5}$ Department of Pharmacy, Buddhist Dalin Tzu Chi General Hospital, Chiayi 62247, Taiwan
}

Correspondence should be addressed to Mei-Ing Chung, meiing.chung@gmail.com

Received 12 August 2010; Accepted 10 April 2011

Academic Editor: Rakesh Joshi

Copyright ( 2011 Yuh-Feng Wang et al. This is an open access article distributed under the Creative Commons Attribution License, which permits unrestricted use, distribution, and reproduction in any medium, provided the original work is properly cited.

\begin{abstract}
Ferrite magnetic nanoparticles $\left(\mathrm{Fe}_{3} \mathrm{O}_{4}\right.$ or iron (II,III) oxide; 15-25 nm of diameter) were developed. These magnetic nanoparticles are a potential vehicle for magnetically induced target aggregation in living animals. In this preliminary study, the radiochemical purity for the radiolabeled magnetic nanoparticles was examined, and the possibility of the magnetically induced targeting of the radio-nanoparticles was evaluated. Our results showed that radiolabeled ferrite nanoparticles can be used as magnetic targeting agents with high labeling efficiency and stability. These particles can be distributed within living animals via intravenous injection, and the biodistribution of the particles can be potentially controlled by external magnetism. These evaluations will be the groundwork for the future development of delivery techniques for radiopharmaceuticals through external magnetic control.
\end{abstract}

\section{Introduction}

Efficient drug delivery to a target organ or tissue achieves the desired therapeutic outcome with fewer side effects. Therefore, the design of a system for discretelytargeted drug delivery would be a therapeutic advance. This idea has been applied extensively for the control of infections and for the management of malignant lesions. Monoclonal antibodies are examples of the initial development of target therapy [14]. They can also serve as drug carriers. Some directedtarget monoclonal antibody therapies are available commercially and have been used in clinical applications $[3,4]$. In addition to the biological characteristics of antigen-antibody affinity, the effective localization of applied reagents using histological characteristics is another approach that has been used [58]. Recently, Y-90 microspheres, which were developed for the treatment of metastatic intrahepatic malignancies, were implanted directly in the capillary network in front of the tumor mass $[9,10]$.
We have developed ferrite magnetic nanoparticles $\left(\mathrm{Fe}_{3} \mathrm{O}_{4}\right.$, or iron (II,III) oxide). These nanoparticles can be radiolabeled with Tc-99m pertechnetate to Tc-99m nanoferrite $[11,12]$. We hypothesize that this radiolabeled magnetic nanoparticle can be redistributed within an animal by changing the external magnetism. If that can be achieved, a magnetically derived, target aggregation of nanoparticlecarried therapy would be attainable.

\section{Materials and Methods}

2.1. Preparation of Ferrite Nanoparticles. Ferrite nanoparticles were synthesized in an aqueous solution of iron chloride and ammonium hydroxide using the protocol we previously described $[11,13]$. In brief, the reaction solution of $\mathrm{FeCl}_{2}$ $(0.05 \mathrm{M}), \mathrm{FeCl}_{3}(0.05 \mathrm{M})$, and a $\mathrm{pH}$-adjusting solution of $\mathrm{NH}_{4} \mathrm{OH}(50 \mathrm{~mL})$ were simultaneously dropped into an open vessel (200 $\mathrm{mL}$ in volume) at room temperature. During the synthesis, the amount of $\mathrm{NH}_{4} \mathrm{OH}$ solution was controlled so 
that the $\mathrm{pH}$ of the aqueous solution was near the neutral condition $(7<\mathrm{pH}<9)$. The ferrite nanoparticles were precipitated in the aqueous solution. X-ray diffraction was performed and confirmed that the average diameter of the nanoparticles was approximately $15-25 \mathrm{~nm}$, deduced by Langevin's formulation.

\subsection{Radiolabeling of the Tc-99m Pertechnetate to the Nanopar-} ticles. After collection of the ferrite nanoparticles from the preparation solution, radiolabeling was initiated. Sodium pertechnetate (Tc-99m pertechnetate) was eluted from an Mo-99/Tc-99m generator (Ultra-Techne Kow; Daiichi Radioisotope Laboratories, LTD., Tokyo, Japan), following instructions provided by the manufacturer. Stannous solution (Amerscan Stannous Agent, Amersham plc, Buckinghamshire, UK) was freshly prepared by carefully adding $10 \mathrm{~mL}$ of normal saline to the kit vial. The final concentration of the stannous fluoride in the stannous solution was $0.4 \mathrm{mg} / \mathrm{mL}$.

Labeling was done by mixing the ferrite nanoparticles with the stannous solution and Tc-99m pertechnetate. We placed $0.5 \mathrm{~mL}$ of the ferrite nanoparticle solution in a sterile tube (BD Vacutainer; Becton, Dickinson and Company, NJ, USA), and then the stannous solution $(0.5 \mathrm{~mL})$ was added by a volumetric pipette. Tc- $99 \mathrm{~m}$ pertechnetate were eluted $(1.11 \mathrm{GBq})$ and counted by a dose calibrator (CRC-15R; Capintec, INC., NJ, USA) and placed in sterile tubes. The ferrite nanoparticle and stannous mixtures were then transferred aseptically to the tubes containing Tc- $99 \mathrm{~m}$ pertechnetate using a syringe and carefully delivering the solution down the side wall of the tubes. Mixing was performed manually by shaking the tubes gently to avoid bubble formation.

2.3. Determining the Optimal Protocol. Ferrite nanoparticles labeled with Tc-99m pertechnetate (hereafter "Tc-99m nanoferrite") was examined in 5 preparation protocols. The compositions were as follows (a) nanoferrite $0.5 \mathrm{~mL}$, stannous solution $0.5 \mathrm{~mL}$, and Tc- $99 \mathrm{~m}$ pertechnetate $370 \mathrm{MBq}$; (b) nanoferrite $0.5 \mathrm{~mL}$, stannous solution $0.5 \mathrm{~mL}$, and $\mathrm{Tc}-$ $99 \mathrm{~m}$ pertechnetate $1.11 \mathrm{GBq}$; (c) nanoferrite $0.5 \mathrm{~mL}$, stannous solution $0 \mathrm{~mL}$, and Tc-99m pertechnetate $370 \mathrm{MBq}$; (d) nanoferrite $0.5 \mathrm{~mL}$, stannous solution $1.0 \mathrm{~mL}$, and Tc- $99 \mathrm{~m}$ pertechnetate $370 \mathrm{MBq}$; (e) nanoferrite $2.0 \mathrm{~mL}$, stannous solution $0.5 \mathrm{~mL}$, and Tc-99m pertechnetate $370 \mathrm{MBq}$.

The radiochemical purity (RCP) and stability were determined by means of instant thin layer chromatography (ITLC) analysis. One drop of the specimen was placed on a silica gel ITLC strip. The strip was developed by acetone, and the radioactivity distribution over the strip was determined with a scintillation counter. Radiochemical purity was calculated as the fraction of radioactivity that remained at the origin and was displayed as \%RCP. After the mixture was prepared, its stability was acquired by sequentially repeating the RCP procedure at $10 \mathrm{~min}, 30 \mathrm{~min}$, $1 \mathrm{hr}, 2 \mathrm{hr}, 4 \mathrm{hr}, 6 \mathrm{hr}$, and $24 \mathrm{hr}$.

2.4. In Vitro Confirmation of the Magnetism Property after Radiolabeling. Tc-99m nanoferrite preparation was placed on a plastic plate. A gamma camera (DST-XLi, General Electric Medical Systems, Buc, France) was used to acquire the scintigraphy, and the images were processed and inspected by the working station (POWERstation SPX, IBM RS6000, Vision 5.2.0, General Electric Medical Systems). The plate was placed on a stable table, and a homogeneous distribution of radioactivity was observed on the monitor. Dynamic acquisition was initiated, and a commercially available magnet was then placed at the sidewall of the plate.

2.5. In Vivo Magnetically Derived Aggregation of Tc-99m Nanoferrite. The rats (Wistar strain, male, weighing from 200 to $250 \mathrm{~g}$ ) were anesthetized with an intraperitoneal injection of pentobarbital $(0.006 \mathrm{mg} / 100 \mathrm{~g})$. A gamma camera (DST-XLi, General Electric Medical Systems, Buc, France), fitted with a low-energy, high-resolution collimator was used. Dynamic image acquisitions were set to 600 frames of 0.4 seconds, followed by a static image at $5 \mathrm{~min}, 10 \mathrm{~min}$, and 15 min after the initiation of the study.

In addition to the normal biodistribution studies, an examination of magnetically-derived biodistribution changes was performed. After the study animal was anesthetized, a magnet was taped in contact with the skin on the lower right abdomen. No sutures were used, and no surgical procedure was performed. Image acquisitions followed the same protocol that was used when there was no external magnetic field applied.

2.6. Ethics. All experiments were performed in accordance with the Animal Protection Act of the Council of Agriculture, and they were approved by the Institutional Animal Care and Use Committee of Dalin Tzu Chi General Hospital.

\section{Results}

3.1. Optimal Preparation Protocol. Five different radiolabeled composites were examined. Figure 1 demonstrates the \% RCP and the stability of the labeled composites. All showed good RCP and stability after labeling, except for protocol C, which had no stannous solution. Protocol C showed much poorer labeling efficiency compared to the other protocols, so it was concluded that preparation protocol C should not be used. Protocol E, which had the highest nanoferrite content, showed the highest \%RCP throughout the study. We also noted that there were no significant differences in the \% RCP based on the content of the stannous solution or Tc-99m pertechnetate while the nanoferrite concentration was fixed (protocols A, B, and D). Protocol E appeared to have the highest \%RCP for the preparation of Tc-99m nanoferrite and was acquired for the following studies.

\subsection{In Vitro Confirmation of the Magnetic Property after Radiolabeling. Figure 2 demonstrates the dynamic change of the radioactivity of the Tc-99m nanoferrite. Initially, the distribution was homogeneous, but, after the magnet was placed adjacent to the sidewall of the plate, a tendency of gradual movement of radioactivity toward the magnet was}




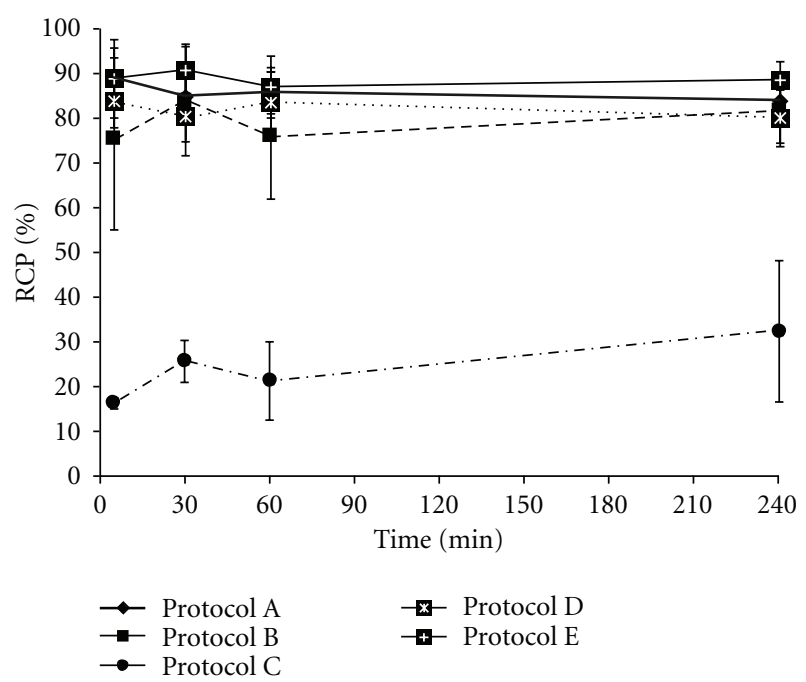

Figure 1: Labeling efficiency and stability test of all the preparation protocols. Protocol A: nanoferrite $0.5 \mathrm{~mL}$, stannous solution $0.5 \mathrm{~mL}$, and Tc- $99 \mathrm{~m}$ pertechnetate $370 \mathrm{MBq}$. Protocol B: nanoferrite $0.5 \mathrm{~mL}$, stannous solution $0.5 \mathrm{~mL}$, and Tc-99m pertechnetate $1.11 \mathrm{GBq}$. Protocol C: nanoferrite $0.5 \mathrm{~mL}$, stannous solution $0 \mathrm{~mL}$, and Tc-99m pertechnetate $370 \mathrm{MBq}$. Protocol D: nanoferrite $0.5 \mathrm{~mL}$, stannous solution $1.0 \mathrm{~mL}$, and $\mathrm{Tc}-99 \mathrm{~m}$ pertechnetate $370 \mathrm{MBq}$. Protocol E: nanoferrite $2.0 \mathrm{~mL}$, stannous solution $0.5 \mathrm{~mL}$, and Tc-99m pertechnetate $370 \mathrm{MBq}$.

observed. Finally, an obvious "hot" focus on the right side of the plate was observed.

3.3. In Vivo Animal Studies. Immediately after intravenous administration of the radiolabeled nanoferrite, rapid distributions of the radioactivity to the lungs, liver, and kidneys were observed (dynamic images not shown). After several hours, the distributions to all of the internal organs diminished, except for the kidneys. The increased distribution of radioactivity to the kidneys demonstrated that the genitourinary pathway was the major excretory pathway for the Tc-99m nanoferrite (Figure 3(a)).

A magnet was placed in contact with the skin in the right lower abdominal area, and the image results were significantly different. In addition to the distribution of the radioactivity to the internal organs of the control animals (for which no magnet was used), the test animals (for which a magnet was placed in contact with the skin of the lower, right abdominal area) showed increased uptakes and sequestration of radioactivity in the area where the magnet was placed. From $5 \mathrm{~min}$ to $15 \mathrm{~min}$ after the initiation of the study, the radioactivity in this area became more and more obvious (Figure 3(b), arrows).

\section{Discussion}

The use of practical application of therapeutics in treating patients is hindered by the lack of efficiency in delivering these agents directly to the target lesions. Targeted drug delivery systems have undergone dramatic development since the first biopharmaceutical products were introduced a few decades ago. Since that time, drug delivery systems with various approaches to target specific areas of the body have been established, and these innovations have been used widely. Targeted drug delivery comprises active and passive targeting [14-16]. The active targeting agents are designed to interact only with the proposed target, such as monoclonal antibodies and RNA interference therapies, which minimizes any adverse effects. On the other hand, in order to be effective, passive targeting agents are designed to be delivered to a specific site by the use of innovative devices such as nasal sprays and transdermal patches for drug delivery. The development of nanoparticle engineering devices, especially nanomedical technologies, is increasing because these systems can help to maximize the bioavailability of drugs at the specific sites where they are needed [17]. The main advantage of passive technologies over active technologies is that it makes it possible to deliver a smaller dose of the drug directly where it is needed.

In this magnetically-directed targeting distribution study, magnetic ferrite nanoparticles were used. In order to have a good in vivo trace of biodistribution and target aggregation, radiolabeling is essential. A series of preparation protocols were examined to determine the optimal ingredients (Figure 1). We found that the \%RCP was directly proportional to the amount of ferrite nanoparticles (protocol $\mathrm{E}$ versus protocol A). The stannous solution is essential in the labeling procedure; without it, the \%RCP was very poor (protocol A versus protocol C). However, no differences were noted from the protocols as a result of the different proportions of the stannous solution. With a fixed concentration of ferrite nanoparticles, neither higher radioisotope content, nor larger quantities of stannous made any significant difference in the RCP (protocol A versus protocol B and protocol A versus protocol D, respectively).

The fact that the magnetic property of the ferrite nanoparticles was not changed as a result of radiolabeling is important. We conducted an in vitro study and demonstrated the specific movement of Tc-99m nanoferrite by an applied magnetic field (Figure 2). In viewing the magnetic-induced targeting effect of the Tc-99m nanoferrite to a living animal, a magnet was placed on the skin of the right lower abdomen of a rat, and the Tc-99m nanoferrite was injected. In comparison with the scintigraphy of the rat without a magnet, a radioactivity-enriched area was found in the corresponding area of the rat where the magnet was located (Figure 3). As a consequence, the magnetically-derived target aggregation of the radiolabeled magnetic nanoparticles was established.

Problems and limitations should be eliminated, such as the control of the external magnetic field. We noticed that the external magnet had to be applied in close proximity to the injection site to obtain a high efficiency of magnetic attraction of the radiolabeled ferrite nanoparticles. When the magnet was placed on the rat's neck, there were no apparent magnetic attraction effects (no image). Efficient external magnetism control and related mechanism studies are issues of importance that need further investigation. In addition, the external magnetism affected the nanoparticles and caused them to aggregate in superficial areas of the rat's body. In order to be valid and useful, the magnetic 


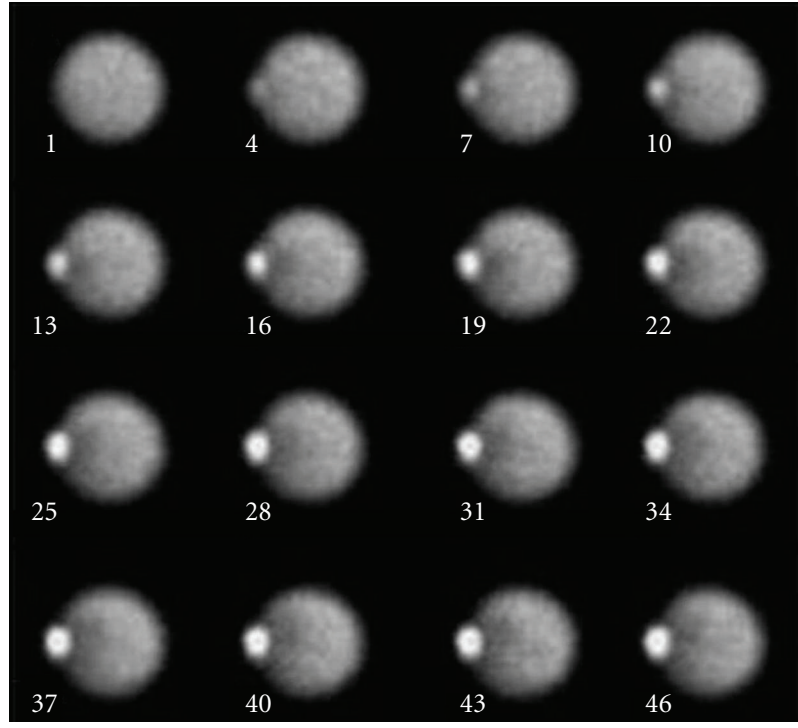

(a)

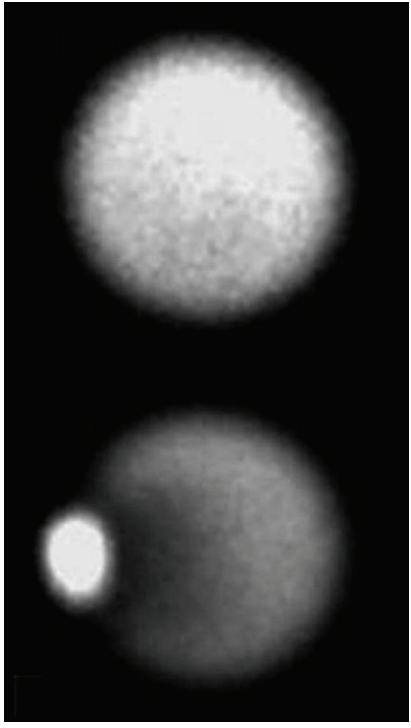

(b)

FIgURE 2: In vitro observation of the magnetically forced distribution of Tc-99m nanoferrite using a gamma camera. These data provide evidence that the physical property of magnetism was not distorted by the process of radiolabeling. (a) Dynamic acquisition showed the tendency of the radioactivity to gradually move toward the magnet. (b) An enlarged picture of the dynamic acquisitions: upper, the initial status; lower, the final image. An obvious "hot" focus on the right side of the plate was exhibited.

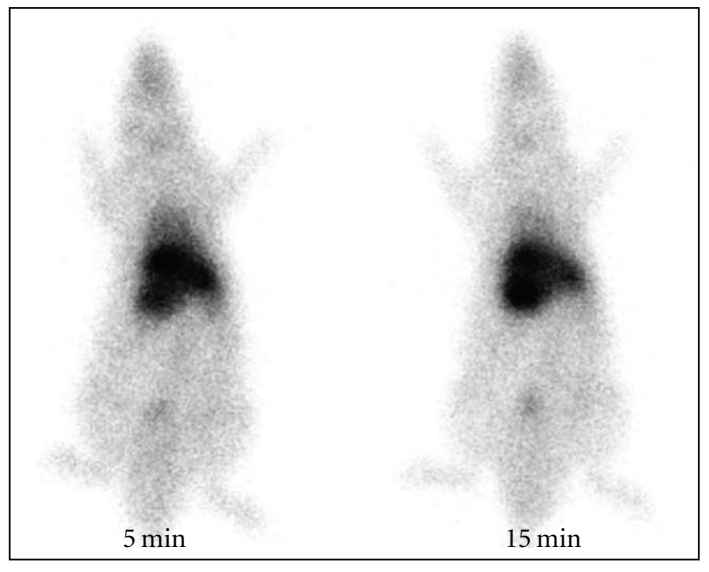

(a)



(b)

FIGURE 3: Biodistribution and magnetically-derived biodistribution changes to the Tc-99m nanoferrite particles. Images were acquired at 5 and $15 \mathrm{~min}$ after injection from the tail vein of the rats, and the acquisition time was 60 seconds. (a) Biodistribution of the Tc-99m nanoferrite; (b) A magnet was placed over the right lower abdominal area of the rat before administration of Tc-99m nanoferrite; an area with increased radioactivity was demonstrated (arrows).

effect should be centralized and deeply applied to the internal organ to direct the approach of the pharmaceuticals. Finally, after the therapeutic purpose has been achieved, the aggregated nanoparticles should be dissociated as rapidly as possible to avoid the formation of thrombosis.

Magnetically radioactive nanoparticles have the advantage of being able to highly concentrate radioactivity in a specific area while minimizing the distribution of the radioactivity to surrounding tissues. Our study showed the possibility of very beneficial usage of radiolabeled ferrite nanoparticles in the future. For example, (1) this model could be a carrier for precise therapeutic reagents, such as a specific tumor agent, an antibiotic, or a regeneration activator, and (2) instead of Tc-99m pertechnetate, a radioisotope with therapeutic effects, such as Re-188, I-131, or Y-90, could be applied. Our results suggest the future development of targeted delivery of radiopharmaceuticals through in vitro control. 


\section{Conclusions}

We have reported a novel preparation of Tc-99m nanoferrite particles. This labeling procedure is achievable as a nuclear medicine laboratory practice. After radiolabeling, these Tc99m nanoferrite particles maintained their magnetic property. This finding was confirmed by in vitro observation, and it was also proven by the results of the in vivo animal study. The radioactivity can be enriched at a specific site by applying an external magnet. Our results imply that these radiolabeled nanoparticles can serve as a vector for magnetically-derived targeting of therapeutic pharmaceuticals.

\section{References}

[1] R. A. Clynes, T. L. Towers, L. G. Presta, and J. V. Ravetch, "Inhibitory Fc receptors modulate in vivo cytoxicity against tumor targets," Nature Medicine, vol. 6, no. 4, pp. 443-446, 2000.

[2] C. A. Reade and A. K. Ganti, "EGFR targeted therapy in nonsmall cell lung cancer: potential role of cetuximab," Biologics, vol. 3, pp. 215-224, 2009.

[3] M. D. Pescovitz, C. J. Greenbaum, H. Krause-Steinrauf et al., "Rituximab, B-lymphocyte depletion, and preservation of beta-cell function," New England Journal of Medicine, vol. 361, no. 22, pp. 2143-2152, 2009.

[4] B. Li, L. Zhao, H. Guo et al., "Characterization of a rituximab variant with potent antitumor activity against rituximabresistant B-cell lymphoma," Blood, vol. 114, no. 24, pp. 50075015, 2009.

[5] M. F. Giblin, G. L. Sieckman, T. D. Shelton, T. J. Hoffman, L. R. Forte, and W. A. Volkert, "In vitro and in vivo evaluation of $177 \mathrm{Lu}-$ and $90 \mathrm{Y}-$ labeled E. coli heat-stable enterotoxin for specific targeting of uroguanylin receptors on human colon cancers," Nuclear Medicine and Biology, vol. 33, no. 4, pp. 481$488,2006$.

[6] Z. Shen, W. Wei, Y. Zhao et al., "Thermosensitive polymerconjugated albumin nanospheres as thermal targeting anticancer drug carrier," European Journal of Pharmaceutical Sciences, vol. 35, no. 4, pp. 271-282, 2008.

[7] J. E. Cyr, D. A. Pearson, D. M. Wilson et al., "Somatostatin receptor-binding peptides suitable for tumor radiotherapy with Re-188 or Re-186. Chemistry and initial biological studies," Journal of Medicinal Chemistry, vol. 50, no. 6, pp. 1354-1364, 2007.

[8] D. Kopelman and M. Papa, "Magnetic resonance-guided focused ultrasound surgery for the noninvasive curative ablation of tumors and palliative treatments: a review," Annals of Surgical Oncology, vol. 14, no. 5, pp. 1540-1550, 2007.

[9] B. Sangro, J. I. Bilbao, M. Iñarrairaegui, M. Rodriguez, P. Garrastachu, and A. Martinez-Cuesta, "Treatment of hepatocellular carcinoma by radioembolization using y microspheres," Digestive Diseases, vol. 27, no. 2, pp. 164-169, 2009.

[10] K. T. Sato, R. J. Lewandowski, M. F. Mulcahy et al., "Unresectable chemorefractory liver metastases: radioembolization with Y microspheres-safety, efficacy, and survival," Radiology, vol. 247, no. 2, pp. 507-515, 2008.

[11] C. M. Fu, Y. F. Wang, Y. C. Chao, S. H. Hung, and M. D. Yang, "Directly labeling ferrite nanoparticles with Tc-99m radioisotope for diagnostic applications," IEEE Transactions on Magnetics, vol. 40, no. 4, pp. 3003-3005, 2004.
[12] C. M. Fu, Y. F. Wang, Y. F. Guo, T. Y. Lin, and J. S. Chiu, "In vivo bio-distribution of intravenously injected Tc-99 m labeled ferrite nanoparticles bounded with biocompatible medicals," IEEE Transactions on Magnetics, vol. 41, no. 10, pp. 4120-4122, 2005.

[13] AI. Y. Wang, C. L. Kuo, J. L. Lin, C. M. Fu, and Y. F. Wang, "Study of magnetic ferrite nanoparticles labeled with Tc- pertechnetate," Journal of Radioanalytical and Nuclear Chemistry, vol. 284, no. 2, pp. 405-413, 2010.

[14] A. Philipp, M. Meyer, and E. Wagner, "Extracellular targeting of synthetic therapeutic nucleic acid formulations," Current Gene Therapy, vol. 8, no. 5, pp. 324-334, 2008.

[15] V. Hofmeister, D. Schrama, and J. C. Becker, "Anti-cancer therapies targeting the tumor stroma," Cancer Immunology, Immunotherapy, vol. 57, no. 1, pp. 1-17, 2008.

[16] K. Manjunath, J. S. Ready, and V. Venkateswarlu, "Solid lipid nanoparticles as drug delivery systems," Methods and Findings in Experimental and Clinical Pharmacology, vol. 27, no. 2, pp. 127-144, 2005.

[17] K. Cho, X. Wang, S. Nie, Z. Chen, and D. M. Shin, “Therapeutic nanoparticles for drug delivery in cancer," Clinical Cancer Research, vol. 14, no. 5, pp. 1310-1316, 2008. 

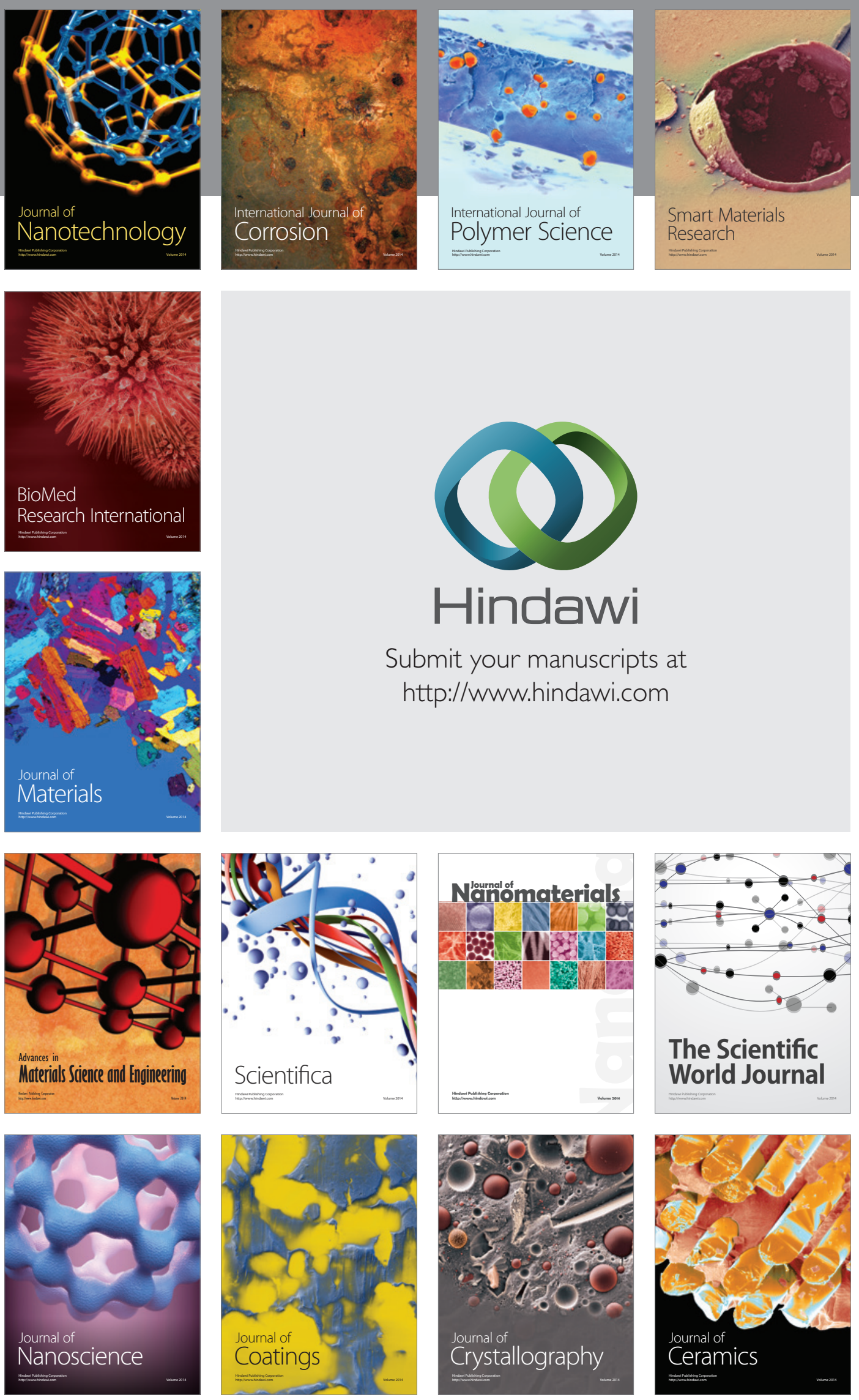

The Scientific World Journal

Submit your manuscripts at

http://www.hindawi.com

\section{World Journal}

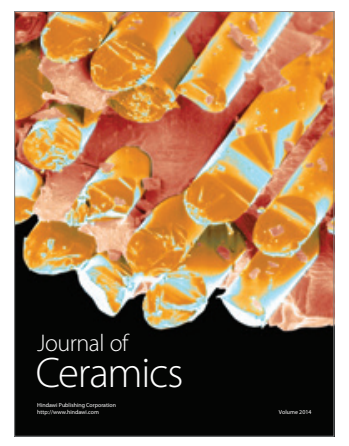

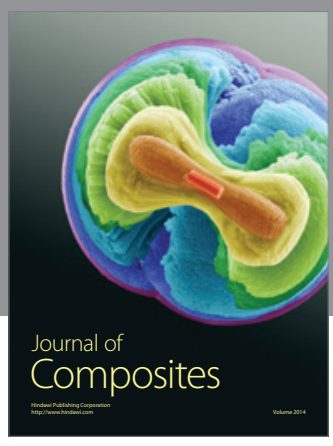
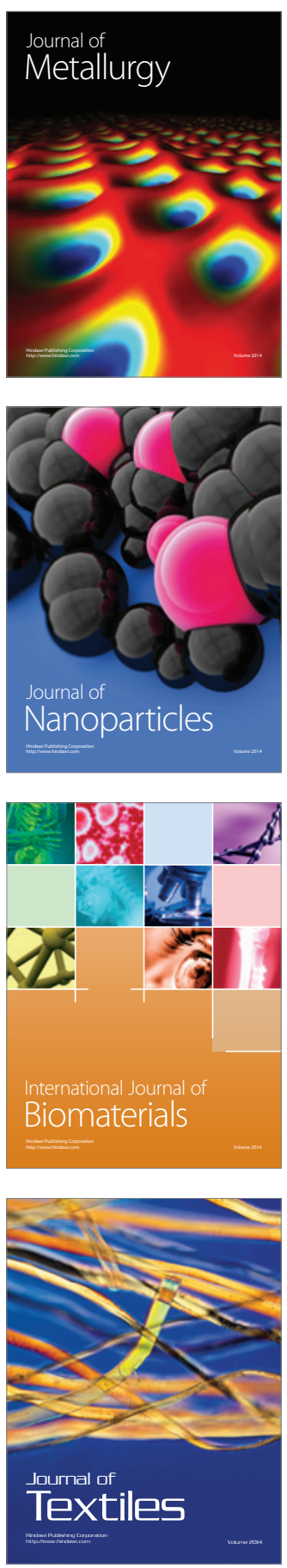